\title{
Devic's neuromyelitis optica associated with active pulmonary tuberculosis: a case report
}

\author{
souheil zayet ${ }^{1}$, Aida Zaghdoudi ${ }^{2}$, Hejer Harrabi ${ }^{2}$, Ahmed Goubantini², and Hanene Tiouiri \\ Benaissa $^{2}$ \\ ${ }^{1}$ Hôpital La Rabta \\ ${ }^{2}$ Hopital La Rabta
}

October 1, 2020

\begin{abstract}
Devic's Optic neuromyelitis (OND) is a rare central nervous system inflammation resulting in optic neuritis and/or myelitis. The discovery of a highly specific serum autoantibody biomarker for the diagnosis has triggered a great interest to conduct further researches regarding this disease. Its association to Tuberculosi (TB) is very rare.

Introduction:

Considered for a long time as a form of multiple sclerosis, Devic's optic neuromyelitis also called Neuromyeliti Optica (NMO) is a rare anatomo-clinical entity that involves optic pathways and spinal cords without important brain injuries. NMO is an inflammatory demyelinating disease of the central nervous system (CNS). Its association to pulmonary tuberculosis is even rarer and has only been reported a few times.
\end{abstract}

Case Report:

We report the case of a 28 year-old male patient without any medical history, who presented to emergency room with eye pain and blurred vision, gait disorders and dysuria.

He also reported a progressive and profound alteration of the general state with fever, and low abundance hemoptysis. In our department, the progression has been towards complete urine retention with the setup of a spastic paraplegia in few days.

Brain and spinal cord Magnetic Resonance Imaging (MRI) was performed, which revealed a cervio-dorsal transversal myelitis extended from C5 to T10 (figure 1) with optic nerve and optic chiasma contrast recording. The lumbar puncture showed a clear cerebrospinal fluid (CSF) with albumin-cytorrachiac dissociation (White Blood Cell (WBC) count of $\left.7 / \mathrm{mm}^{3}\right)$, elevated protein concentration $(3.23 \mathrm{~g} / \mathrm{l}$; normal $<0.45 \mathrm{~g} / \mathrm{l}$ ) and low glycorrhachia (ratio of $1 / 3$ ).

Direct examination and CSF culture were defective. Detection of Herpes Simplex Virus (HSV), Varicella Zoster Virus (VZV), enteroviruses, Listeria monocytogenes and Mycobacterium tuberculosis by Polymerase Chain Reaction (PCR) technique in the CSF was negative. Anti-NMO or anti-aquaporin 4 (AQP4) antibodies in the CSF was positive.

Ophthalmological examination showed the presence of bilateral papillary edema. Visual-evoked potential indicated a significant prolongation of the latency of the P100 wave.

Chest X-ray showed a bilateral micronodular infiltrate (figure 2) . A thoracic Computed Tomography (CT) scan revealed multiple lung injuries with bilateral acinar nodes, prevailing in the upper left lobe area associated with several cavitary lesions. 
Tuberculin skin test (TST/Mantoux) was negative. Direct examination of the sputum did not reveal any acid-resistant bacillus (ARB). Pulmonary tuberculosis was diagnosed via endotracheal aspirate culture results. Mycobacterium tuberculosis was found in the culture. HIV serology was negative.

The diagnosis of Devic's syndrome was based on the criteria of Wingerchuk and al (1) revealing associated pulmonary tuberculosis. Parenteral corticosteroid therapy was prescribed with immunoglobulins. Antitubercular therapy (ATT) was associated for 6 months. The outcome was only partially favorable with persistent paraplegia.

Discussion

OND is characterized by the association of optic damage and myelopathy due to acute demyelination of optic nerves and spinal cord caused by a dysimmunitary mechanism (2).

During OND, the immune system produces autoantibodies that target a protein called AQP4. It is a protein of the central nervous system expressed by astrocytes. Thereafter a cascade of reactions leading to the damage of the entire nervous tissue is triggered.

These reactions cause an inflammation leading to the destruction of the myelin sheath covering the neurons but also the neuron itself. This process explains the neurological symptoms of the disease.

Although the association of OND to active pulmonary tuberculosis has been reported and is not a simple coincidence, a definite affiliation between the two conditions has not been demonstrated (3).

This association only can be established after having excluded a pulmonary and neurological localisation of the TB. The pathophysiological mechanism is still uncertain.

The study of the spinal cord injuries of autopsied patients has demonstrated the role of humoral immunity dysregulation, triggered by an infectious disease. Indeed, Mycobacterium tuberculosis surface antigens might initiate the production of reactive antibodies against aquaporin-4 proteins. This process would lead to an acute demyelination induced by BK in our case.

The onset of demyelination clusters far from infectious clusters in tuberculous encephalitis and experimental studies of demyelination after intracisternal injection of antigenic BK or tuberculin extracts are also arguments supporting the dysimmunitary mechanism.

However, since the immunitary response against active TB infection is commonly set towards cell-mediated immune response rather than a humoral response, the involvement of this mechanism is still not proven (4).

Another hypothesis that has been discussed is the anti-bacillary treatment toxicity, but this remains inconclusive considering the possibility of neuro-ophthalmological damage before the initiation of TB treatment.

Moreover, a clinical trial carried in China in patients suffering from OND have concluded to a beneficial impact of anti-TB treatment in patients suffering from steroid-refractory OND, even if the tuberculosis was not patent (4).

The prevailing of pulmonary localization is due to its bacillary population richness compared to other sites. Nevertheless, other localizations of TB have been reported such as abdominal localization (5).

Also, the first case of NMO associated to TB was a tuberculous nephritis reported in a 41-year-old woman in Nigeria (6).

As neurologic deficits are mainly due to an inflammatory process, these injuries respond to medical therapy alone without any surgical intervention if diagnosed promptly (7).

The treatment is based on corticosteroids and antituberculous therapy. A partial recovery of motor symptoms and sphincter control with permanent blindness has been reported in the majority of the cases (8). 
The long-term clinical efficiency of ATT in patients with steroid-refractory NMO has been proved. This treatment can reduce disease's activity and progression resulting in a significant recovery of neurological deficits (9).

We insist on the fact that NMO like disorders are an important diagnosis to consider in patients treated for active TB who present with acute neurological disorders such as transverse myelitis and/or optic neuritis. Thus, Serum anti-Aqp-4 antibodies should be part of the diagnostic tests in these patients (3).

An immune dysfunction caused by Mycobacterium tuberculosis seems to be the most likely hypothesis explaining the association of pulmonary TB to OND in terms of pathophysiology.

Whenever demyelinating spinal cord injury in combination with optic pathways damage is diagnosed in a case of tuberculosis we must first rule out brain injuries by direct mechanism before concluding to Devic's syndrome.

\section{Acknowledgment: None}

External funding: None

Conflict of interest : All authors declare that there is no conflict of interest.

\section{Authors' Contribution :}

1. Substantial contributions to conception, analysis and interpretation of data; Souheil Zayet, Aida Zaghdoudi

2. Drafting the manuscript and revising it critically for important intellectual content : Souheil Zayet, Aida Zaghdoudi , Hejer Harrabi

3. Final approval of the version to be published : All authors

4. Agreed to be accountable for all aspects of the work in ensuring that questions related to the accuracy or integrity of any part of the work are appropriately investigated and resolved : All authors

References

1. Wingerchuk DM, Banwell B, Bennett JL, Cabre P, Carroll W, Chitnis T, et al. International consensus diagnostic criteria for neuromyelitis optica spectrum disorders. Vol. 85, Neurology. Lippincott Williams and Wilkins; 2015. p. 177-89.

2. Zhong X, Zhou Y, Lu T, Wang Z, Fang L, Peng L, et al. Infections in neuromyelitis optica spectrum disorder. Vol. 47, Journal of Clinical Neuroscience. Churchill Livingstone; 2018. p. 14-9.

3. Zatjirua V, Butler J, Carr J, Henning F. Neuromyelitis optica and pulmonary tuberculosis: A case-control study. Int J Tuberc Lung Dis. 2011 Dec;15(12):1675-9.

4. Sridhar S, Chan JFW, Yuen KY. Fatal anti-aquaporin-4 seropositive neuromyelitis optica spectrum disorder in tuberculosis. BMC Infect Dis. 2014 Aug 28;14(1).

5. Bhatty SA, Lal H, Talib A, Mahmood K, Naqvi I, Zaidi SS. Neuromyelitis Optica (NMO) with abdominal tuberculosis (TB). J Coll Physicians Surg Pakistan. 2015 Oct 1;25:S102-4.

6. Iorio R, Monforte M, Pierconti F, Iodice F, Sabatelli M. Tuberculous nephritis accompanying neuromyelitis optica: Causal or coincidental association? Vol. 261, Journal of Neurology. Dr. Dietrich Steinkopff Verlag GmbH and Co. KG; 2014. p. 1028-30.

7. Sahu SK, Giri S, Gupta N. Longitudinal extensive transverse myelitis due to tuberculosis: A report of four cases. J Postgrad Med. 2014 Oct 1;60(4):409-12.

8. Jarius S, Wildemann B, Paul F. Neuromyelitis optica: Clinical features, immunopathogenesis and treatment. Clin Exp Immunol. 2014 May;176(2):149-64. 
9. Feng YQ, Guo N, Huang F, Chen X, Sun QS, Liu JX. Anti-tuberculosis treatment for Devic's neuromyelitis optica. J Clin Neurosci. 2010 Nov;17(11):1372-7.

Figures legends:

Figure 1: Spinal cord Magnetic Resonance Imaging (sagittal T2 sequence): A transverse cervico-dorsal myelitis extended from C5 to T10 with multifocal contrast enhancements

Figure 2: Face chest X-ray: Bilateral micro nodular infiltrate

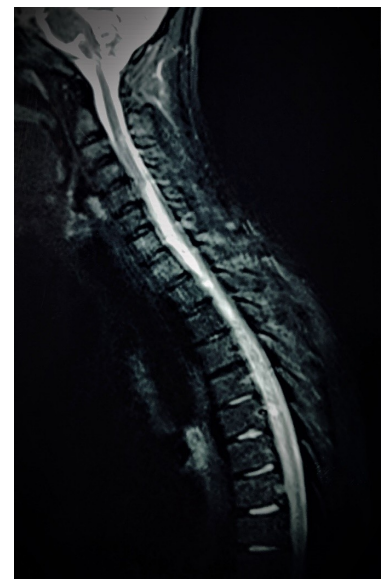

Figure 1

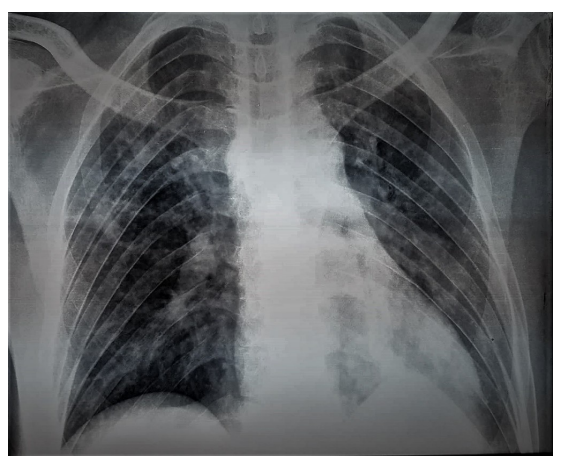

Figure 2 